\title{
Resistance of Pepper Cultivars and Accessions of Capsicum spp. to Sclerotinia sclerotiorum
}

\author{
Y. Yanar and S. A. Miller, Department of Plant Pathology, The Ohio State University, Ohio Agricultural Research \\ and Development Center, Wooster, OH 44691
}

\begin{abstract}
Yanar, Y., and Miller, S. A. 2003. Resistance of pepper cultivars and accessions of Capsicum spp. to Sclerotinia sclerotiorum. Plant Dis. 87:303-307.

Sclerotinia stem and fruit rot, caused by Sclerotinia sclerotiorum (Lib.) de Bary, is a potentially serious disease of bell peppers (Capsicum annuum L.), affecting both seedlings and mature plants. Management options for the disease are limited and information is not available on resistance in Capsicum spp. to this pathogen. The level of resistance of 12 commercial pepper cultivars and 110 Capsicum spp. accessions to Sclerotinia sclerotiorum was determined by using limited-term and ascospore inoculation methods. None of the commercial cultivars tested were resistant to Sclerotinia stem rot. Stem rot incidence among the Capsicum spp. accessions tested by ascospore inoculation ranged from 0 to $100 \%$. Fifty-eight accessions had significantly less stem disease than the susceptible commercial cultivar Marengo. There was no correlation between results of the limited-term and ascospore inoculation tests. These results demonstrate for the first time that a high level of Sclerotinia stem rot resistance exists among the Capsicum spp. accessions. Several accessions may be suitable for use in breeding programs to increase resistance in commercial pepper cultivars.
\end{abstract}

Sclerotinia sclerotiorum (Lib.) de Bary causes disease on a wide range of herbaceous plant species (7), including bell pepper (Capsicum annuum L.). Serious losses in pepper attributed to Sclerotinia stem and fruit rot have been documented in Ohio (25) and elsewhere (4-6,16$18,21,23)$. In Ohio, the disease was first documented in the southeastern part of the state (Gallia County) during 1992. Incidence of stem rot in pepper seedlings 10 days after transplanting ranged from 10 to $20 \%$ in the affected field. The disease also occurred later in the season on flowering plants and fruit. In August 1992, an estimated 30 to $40 \%$ loss due to stem and fruit rot was observed in a 20 -acre processing pepper field in Henry County (25). In a 1996 survey of eight pepper fields in northwest Ohio, incidence of stem rot in seedlings ranged from 0 to $26 \%$ (24). Stem rot of pepper and tomato seedlings grown on raised beds covered with plastic mulch

Corresponding author: S. A. Miller

E-mail: miller.769@osu.edu

This study was supported by The Ohio State University, OARDC, Gaziosmanpasa University (Tokat, Turkey), and the Ohio Vegetable and Small Fruit Research and Development Program.

Current address of first author: Gaziosmanpasa University Faculty of Agriculture, Department of Plant Protection, Tokat, Turkey

Accepted for publication 14 October 2002.

Publication no. D-2002-1230-03R

(C) 2003 The American Phytopathological Society was observed in at least two counties in northern Ohio during 2001 and 2002 (S. Miller, unpublished).

Control of $S$. sclerotiorum is difficult under cool, moist environmental conditions, which are common in Ohio at the beginning and end of the pepper-growing season. Currently, no fungicides are registered for control of this disease on peppers. In addition, the ability of sclerotia of the fungus to survive in the soil for three or more years (1) and the wide host range of the fungus make cultural control difficult, emphasizing the need for resistant cultivars. The level of resistance of commercial cultivars is unknown and potential sources of resistance to the pathogen in $C$. annuиm and other Capsicum spp. have not been identified.

The purpose of this study was to determine the level of resistance to $S$. sclerotiorum in commercial pepper cultivars under growth chamber conditions and to identify sources of resistance to the pathogen among Capsicum spp. accessions.

\section{MATERIALS AND METHODS}

Seedling production. Seed of 110 accessions of Capsicum spp. (Table 1) supplied by the Plant Genetic Resources Conservation Unit (Griffin, GA) and $12 C$. annuum cultivars from commercial seed companies (Table 2) were sown in the greenhouse. Seed were planted in 128-cell black plastic flats (Landmark Plastic Corp., Akron, OH) filled with Baccto High Density Professional Planting Mix (Michigan Peat Co., Houston, TX) and watered daily or as needed. Flats were placed on greenhouse benches where the air temperature was maintained at $28 \pm 2{ }^{\circ} \mathrm{C}$ to facilitate seed germination. Seed typically germinated within 7 to 10 days. After the first true leaf was fully expanded, two or three seedlings were transplanted into 15cm-diameter plastic pots containing a steamed greenhouse soil mixture consisting of $43 \%$ each of Wooster silty-loam and muck soil and $14 \%$ peat moss, to which lime at $1.2 \mathrm{~kg} / \mathrm{m}^{3}$ was added. Pots were fertilized weekly with Peter's 20-20-20 (N$\mathrm{P}-\mathrm{K})$ standard fertilizer (454 g/378 liters of water) and watered once per day. Temperature in the greenhouse was adjusted to $24 \pm 2^{\circ} \mathrm{C}$ during the day and $18 \pm$ $2{ }^{\circ} \mathrm{C}$ at night.

Sclerotia production. Isolates $\mathrm{Ss} 3$ and Ss6 of S. sclerotiorum, recovered from naturally diseased pepper plants collected in northwest Ohio in 1992 and maintained on potato dextrose agar (PDA), were used to produce sclerotia. Disks (5-mm) from the margin of actively growing colonies were transferred to polystyrene petri plates (15 by $100 \mathrm{~cm}$ ) containing $20 \mathrm{ml}$ of PDA. Cultures were grown at $20^{\circ} \mathrm{C}$ until mycelia approached the edge of the plate; then, the contents of one plate were chopped and spread on a single contiguous layer of carrot disks per aluminum foil tray (41.9 by 30.5 by $6.4 \mathrm{~cm}$; Handi-Foil Co., Wheeling, IL) previously autoclaved at $121^{\circ} \mathrm{C}$ for 30 min on each of two consecutive days. Inoculated carrot disks were covered with sterilized aluminum foil and incubated on a laboratory bench at $20 \pm 2^{\circ} \mathrm{C}$. Mycelia completely covered the surface of carrot pieces within 5 days and sclerotial initials were observed within 10 to 12 days. Sclerotia were harvested 4 weeks after the cultures were seeded by pouring the mycelia, sclerotia, and remains of carrot tissue into a No. 14 soil sieve (12-mesh) and washing under tap water to separate the sclerotia from other materials. Sclerotia that were obtained were dried on paper towels overnight at $22 \pm 2{ }^{\circ} \mathrm{C}$ and stored in plastic bags at $4^{\circ} \mathrm{C}$ until used.

Ascospore production. Sclerotia produced on autoclaved carrot discs were surface sterilized in $95 \%$ ethanol for $90 \mathrm{~s}$ as described by Huang and Kozub (10) and air dried for $15 \mathrm{~min}$ in a laminar flow hood. Twenty sclerotia were placed in 9$\mathrm{cm}$-diameter glass petri plates containing $15 \mathrm{ml}$ of sterile distilled water. Plates were wrapped with parafilm and conditioned at $4^{\circ} \mathrm{C}$ for 6 weeks in the dark. Then they 
were incubated at $15^{\circ} \mathrm{C}$ under fluorescent light $\left(47.5 \mathrm{E} \mathrm{s}^{-1} \mathrm{~m}^{-2}\right)$ for $14 \mathrm{~h}$ followed by $10 \mathrm{~h}$ of dark/day. Stipes formed on sclerotia 4 weeks after transferring to $15^{\circ} \mathrm{C}$, and mature apothecia developed after 1 to 2 weeks. Ascospores were collected from mature apothecia directly on nucleopore polycarbonate filters (type GS, $47 \mu \mathrm{m}$; Thomas Scientific, Swedesboro, NJ) as described by Hunter et al. (11) and Steadman et al. (19). Filters with ascospores were stored in glass jars containing calcium chloride $\left(\mathrm{CaCl}_{2}\right)$ as a desiccant at -19 or $4^{\circ} \mathrm{C}$ until used.

Ascospore inoculation. Nucleopore filters containing ascospores of isolate Ss6 of $S$. sclerotiorum were back washed with distilled water and the final ascospore concentration was adjusted to $5 \times 10^{4}$ spores $/ \mathrm{ml}$. The suspension was sprayed onto three 8-week-old pepper seedlings with a hand sprayer. Control plants were sprayed with distilled water only. Three replicate pots for each cultivar or accession were arranged in a randomized complete block design on the upper and lower shelves of steel mesh benches in a growth chamber at $20^{\circ} \mathrm{C}$ with a $12-\mathrm{h}$ photoperiod. Relative humidity of 95 to $100 \%$ was maintained by using two humidifiers (Humidifier series 500; Hermidifier Co. Inc. Lancaster, PA) per 2.45-by-1.90-by-2.12$\mathrm{m}$ growth chamber. Beginning 4 days after inoculation, the number of infected stems and dead plants in each pot was recorded every other day for 15 days. Disease incidence was expressed as a percentage of the total number of diseased and dead plants in each accession or cultivar 15 days after inoculation, and disease severity was expressed as the area under the disease progress curve (AUDPC; 8). This experiment was conducted three times.

Limited-term inoculation. Limitedterm inoculation (LTI) was performed as described by Hunter et al. (12), except that PDA disks colonized with mycelia of $S$. sclerotiorum were used to provide a consistent amount of inoculum to each plant. Isolate Ss6 of $S$. sclerotiorum was grown on PDA for 2 days at $20^{\circ} \mathrm{C}$; then, 1-cm-diameter disks were cut from actively growing colony margins with a cork borer. A single disk was attached to the stem of each 8-week-old plant (two disks per $10-\mathrm{cm}$-diameter pot) above the cotyledonary node by wrapping it with moist cotton pieces. PDA disks were used for noninoculated control plants. There were three replicate pots per cultivar or accession arranged in a randomized complete block design in a humid growth chamber as described above for ascospore inoculations. Inoculum was removed after $24 \mathrm{~h}$ and plants were maintained under the same conditions for an additional 4 days. Disease incidence was determined 5 days after inoculation by counting the number of
Table 1. Reaction of 110 accessions of Capsicum spp. and the commercial pepper cultivar Marengo tested for seedling resistance to Sclerotinia stem rot by ascospore inoculation

\begin{tabular}{|c|c|c|c|c|}
\hline \multirow[b]{2}{*}{ Capsicum spp., accession } & \multirow[b]{2}{*}{ Source } & \multicolumn{2}{|c|}{ Stem $\operatorname{rot}(\%)^{x}$} & \multirow[b]{2}{*}{ AUDPC } \\
\hline & & Nontrans & Arcsine & \\
\hline \multicolumn{5}{|l|}{ C. аппиит } \\
\hline PI 294452 & Virgin Islands & 92 & 128 & 31 \\
\hline PI 385961 & Kenya & 85 & 117 & 32 \\
\hline Marengo & Seminis Seed & 85 & 117 & 28 \\
\hline PI 401729 & Brazil & 84 & 116 & 30 \\
\hline PI 390971 & Israel & 81 & 112 & 29 \\
\hline PI 164564 & Spain & 81 & 112 & 22 \\
\hline PI 124080 & India & 81 & 112 & 20 \\
\hline PI 401728 & Brazil & 77 & 107 & 29 \\
\hline PI 338490 & Bulgaria & 77 & 107 & 27 \\
\hline PI 164565 & Spain & 74 & 104 & 25 \\
\hline PI 159265 & United States & 74 & 104 & 17 \\
\hline PI 164678 & India & 73 & 102 & 26 \\
\hline PI 281330 & Costa Rica & 73 & 102 & 20 \\
\hline PI 281413 & Peru & 73 & 102 & 31 \\
\hline PI 286419 & Nepal & 70 & 99 & 26 \\
\hline PI 291999 & Israel & 70 & 99 & 25 \\
\hline PI 385960 & Kenya & 59 & 88 & 30 \\
\hline PI 102882 & China & 59 & 88 & 15 \\
\hline PI 105338 & China & 66 & 95 & 26 \\
\hline PI 213915 & Bolivia & 66 & 95 & 21 \\
\hline PI 338974 & Turkey & 66 & 95 & 20 \\
\hline PI 169110 & Turkey & 66 & 95 & 19 \\
\hline PI 251622 & Yugoslavia & 66 & 95 & 18 \\
\hline PI 167062 & Turkey & 66 & 95 & 15 \\
\hline PI 184037 & Yugoslavia & 62 & 91 & 22 \\
\hline PI 263106 & Russia & 59 & 88 & 22 \\
\hline PI 275011 & Bulgaria & 59 & 88 & 20 \\
\hline PI 169106 & Turkey & 55 & 84 & 17 \\
\hline PI 167215 & Turkey & 55 & 84 & 16 \\
\hline PI 390437 & Ecuador & 55 & 84 & 16 \\
\hline PI 123164 & India & 55 & 84 & 16 \\
\hline PI 281400 & Nicaragua & 55 & 84 & 15 \\
\hline PI 124540 & India & 55 & 84 & 11 \\
\hline PI 164961 & Turkey & 55 & 84 & 11 \\
\hline PI 281416 & Philippines & 51 & 80 & 18 \\
\hline PI 275009 & Bulgaria & 51 & 80 & 18 \\
\hline PI 263073 & Russia & 59 & 88 & 16 \\
\hline PI 123469 & India & 59 & 88 & 19 \\
\hline PI 159278 & United States & 48 & 77 & 16 \\
\hline PI 184039 & Yugoslavia & 48 & 77 & 16 \\
\hline PI 249907 & Portugal & 48 & 77 & 14 \\
\hline PI 262905 & Spain & 48 & 77 & 13 \\
\hline PI 169112 & Turkey & 48 & 77 & 10 \\
\hline PI 131352 & Hungary & 44 & 73 & 14 \\
\hline PI 390436 & Ecuador & 44 & 73 & 14 \\
\hline PI 164562 & Spain & 44 & 73 & 11 \\
\hline PI 289762 & Turkey & 37 & 65 & 10 \\
\hline PI 162608 & China & 37 & 65 & 16 \\
\hline PI 169102 & Turkey & 37 & 65 & 13 \\
\hline PI 123166 & India & 37 & 65 & 11 \\
\hline PI 127445 & Afghanistan & 37 & 65 & 10 \\
\hline PI 103048 & China & 37 & 65 & 9 \\
\hline PI 138561 & Iran & 37 & 65 & 7 \\
\hline PI 164561 & Spain & 33 & 61 & 11 \\
\hline PI 257049 & Colombia & 33 & 61 & 10 \\
\hline PI 273429 & United States & 33 & 61 & 10 \\
\hline PI 390970 & Israel & 33 & 61 & 9 \\
\hline PI 135826 & Afghanistan & 33 & 61 & 6 \\
\hline PI 102881 & China & 29 & 57 & 11 \\
\hline PI 148626 & Iran & 29 & 57 & 7 \\
\hline PI 257187 & Peru & 26 & 54 & 11 \\
\hline PI 162607 & China & 26 & 54 & 5 \\
\hline PI 297456 & Spain & 22 & 49 & 5 \\
\hline \multirow[t]{2}{*}{ PI 263074} & Russia & 15 & 40 & 4 \\
\hline & & & \multicolumn{2}{|c|}{ (continued on next page) } \\
\hline
\end{tabular}

${ }^{\mathrm{x}}$ Nontrans $=$ nontransformed and Arcsine $=$ arcsine-transformed. Analysis of variance for percent stem rot was performed on arcsin-transformed data.

${ }^{y}$ AUDPC $=$ area under the disease progress curve.

${ }^{\mathrm{z}} \mathrm{LSD}=$ least significant difference between means, calculated using Fisher's protected least significant difference test $(P \leq 0.05)$. 
collapsed plants. Results were expressed as a percentage of collapsed seedlings in each accession or cultivar. This experiment was repeated twice.

Statistical analyses. Data from the three experiments for each inoculation technique were averaged and transformed using arcsin transformation prior to analysis. Transformed data were analyzed using analysis of variance (ANOVA). Differences between treatment means were detected using Fisher's protected least significant difference test $(P \leq$ 0.05). The AUDPCs for percent stem rot and plants killed were computed by the method of Campbell and Madden (8). Linear regression analysis was used to determine the relationship between ascospore inoculation and LTI methods. All analyses were performed using a SAS statistical package (release 6.04; SAS Institute, Cary, NC).

Table 1. (continued from preceding page)

\begin{tabular}{|c|c|c|c|c|}
\hline \multirow[b]{2}{*}{ Capsicum spp., accession } & \multirow[b]{2}{*}{ Source } & \multicolumn{2}{|c|}{ Stem rot $(\%)^{x}$} & \multirow[b]{2}{*}{ AUDPCy } \\
\hline & & Nontrans & Arcsine & \\
\hline \multicolumn{5}{|l|}{ C. аппиит } \\
\hline PI 159255 & United States & 11 & 34 & 4 \\
\hline PI 281327 & Colombia & 0 & 0 & 0 \\
\hline \multicolumn{5}{|c|}{ C. annuum var. glabriusculum } \\
\hline PI 511887 & Mexico & 100 & 157 & 36 \\
\hline PI 511886 & Mexico & 11 & 34 & 6 \\
\hline \multicolumn{5}{|l|}{ C. baccatum } \\
\hline PI 273425 & United States & 88 & 122 & 32 \\
\hline PI 370005 & India & 66 & 95 & 28 \\
\hline PI 370004 & India & 66 & 95 & 25 \\
\hline PI 200729 & Guatemala & 48 & 77 & 18 \\
\hline PI 439364 & Brazil & 44 & 73 & 15 \\
\hline PI 439361 & Bolivia & 44 & 73 & 8 \\
\hline PI 439360 & Bolivia & 33 & 61 & 10 \\
\hline PI 413669 & Colombia & 33 & 61 & 9 \\
\hline PI 260434 & Bolivia & 11 & 34 & 4 \\
\hline PI 439396 & Peru & 7 & 27 & 1 \\
\hline \multicolumn{5}{|l|}{ C. baccatum var. baccatum } \\
\hline PI 260533 & Brazil & 100 & 157 & 42 \\
\hline PI 260567 & Bolivia & 77 & 107 & 28 \\
\hline PI 238061 & Bolivia & 55 & 84 & 19 \\
\hline PI 215699 & Peru & 48 & 77 & 16 \\
\hline PI 281306 & Bolivia & 33 & 61 & 8 \\
\hline \multicolumn{5}{|l|}{ C. baccatum var. pendulum } \\
\hline PI 241679 & Chile & 59 & 88 & 17 \\
\hline PI 203522 & Mexico & 58 & 87 & 18 \\
\hline PI 152234 & Peru & 51 & 80 & 15 \\
\hline PI 257122 & Colombia & 44 & 73 & 11 \\
\hline PI 188803 & Philippines & 40 & 68 & 12 \\
\hline PI 159235 & United States & 33 & 61 & 7 \\
\hline PI 241674 & Ecuador & 33 & 61 & 7 \\
\hline PI 199506 & Guyana & 22 & 49 & 7 \\
\hline PI 238062 & Brazil & 18 & 44 & 7 \\
\hline PI 224440 & Costa Rica & 7 & 27 & 2 \\
\hline PI 152217 & Peru & 4 & 20 & 2 \\
\hline \multicolumn{5}{|c|}{ C. baccatum var. praetermissum } \\
\hline PI 260595 & Brazil & 22 & 49 & 5 \\
\hline \multicolumn{5}{|l|}{ C. chacoense } \\
\hline PI 260431 & Bolivia & 100 & 157 & 31 \\
\hline PI 260430 & Bolivia & 77 & 107 & 26 \\
\hline PI 260427 & Argentina & 63 & 92 & 15 \\
\hline PI 260426 & Argentina & 40 & 68 & 12 \\
\hline \multicolumn{5}{|l|}{ C. chinense } \\
\hline PI 209589 & Cuba & 88 & 122 & 36 \\
\hline PI 152225 & Peru & 77 & 107 & 26 \\
\hline PI 194879 & Guyana & 55 & 84 & 18 \\
\hline PI 152452 & Brazil & 51 & 80 & 16 \\
\hline PI 152222 & Peru & 33 & 61 & 8 \\
\hline \multicolumn{5}{|l|}{ C. frutescens } \\
\hline PI 159238 & United States & 85 & 117 & 30 \\
\hline PI 188479 & Vanuatu & 63 & 92 & 27 \\
\hline PI 123474 & India & 33 & 61 & 7 \\
\hline \multicolumn{5}{|l|}{ C. galapagoense } \\
\hline PI Grif 1567 & Netherlands & 0 & 0 & 0 \\
\hline \multicolumn{5}{|l|}{ C. pubescens } \\
\hline PI 235047 & Costa Rica & 26 & 54 & 9 \\
\hline PI 585266 & Ecuador & 22 & 49 & 6 \\
\hline \multicolumn{5}{|l|}{ Tubocapsicum anomalum } \\
\hline PI 501532 & Japan & 44 & 73 & 15 \\
\hline $\mathrm{LSD}^{\mathrm{z}}$ & $\ldots$ & $\ldots$ & 36 & 11 \\
\hline
\end{tabular}

\section{RESULTS}

Capsicum spp. accessions. Disease incidence varied from 0 to $100 \%$ among the 110 accessions inoculated with ascospores (Table 1). C. annuum accession PI 281327 and C. galapagoense accession PI Grif 1567 did not develop stem symptoms. Of the 110 accessions tested, 58 had significantly lower incidence of stem rot than the susceptible cultivar Marengo $(85 \%)$. These 58 accessions represented seven different Capsicum spp. and Tubocapsicum anomalum. Thirteen accessions (11 C. annuum, one $C$. chacoense, and one C. baccatum var. pendulum) had slower disease progress than Marengo, but did not differ from Marengo based on percent stem rot 15 days after inoculation. Accessions PI 159255 and PI 281327 (C. annuum), PI 260434 and PI 439396 (C. baccatum), PI 511886 (C. annuum var. glabriusculum), PI 224440 and PI 152217 (C. baccatum var. pendulum), and PI Grif 1567 (C. galopagoense) had $11 \%$ or less stem rot and were significantly more resistant than the 70 accessions with 44 to $100 \%$ stem disease incidence. There was a positive correlation between stem rot incidence and number of plants killed $\left(R^{2}=0.89\right)$ and between AUDPCs for stem rot incidence and plants killed $\left(R^{2}=0.94\right)$. Therefore, only data from stem rot are presented in Table 1.

All of the 12 commercial cultivars screened in the growth chamber using the ascospore inoculation method were susceptible to stem rot (Table 2). Big Bertha was the most susceptible cultivar among those evaluated, while SS 860 was significantly less susceptible than the six most susceptible cultivars (Big Bertha, Astrione, Camelot, Lilac, Pax 2557, and Marengo). Slow disease progress was observed on Tequila, although the final percentage stem rot was not significantly different than that for all but the most susceptible cultivar (Big Bertha).

For commercial pepper cultivars and susceptible Capsicum spp. accessions inoculated with ascospores, the earliest symptom, which usually appeared 3 to 4 days after inoculation, was the development of local brown spots on the leaves. Then brownish, irregularly shaped watersoaked lesions were produced. Bud rot also was observed. Leaf lesions progressed to the petioles and expanded up and down the main stem. Subsequently, the seedlings died, usually within 10 to 15 days. White fluffy mycelial growth was observed in the colonized tissues. Almost all stem infection originated from the node, where the petiole of the infected leaf was attached to the stem.

Correlation was poor between the LTI method and the ascospore inoculation method $\left(R^{2}=0.008\right.$; Fig. 1$)$. Of the 51 accessions tested using the LTI method, 20 had an incidence of stem rot that was within $15 \%$ of plants inoculated with ascospores (Table 3). Fifteen accessions had an 
incidence of stem rot that was 16 to $45 \%$ different between the two tests. The remaining accessions differed in incidence of stem rot in the two tests by more than $45 \%$. Two accessions (PI 152217 and PI Grif 1567) were highly resistant in ascospore inoculation tests but highly susceptible when LTI was used. Two accessions (C. annиum PI 159255 and C. annuum var. glabriusculum PI 511886) were highly resistant when inoculated using either method.

All of the plants in the 12 commercial cultivars inoculated using the LTI method were dead at the end of the 5-day incubation period in each of three tests.

\section{DISCUSSION}

Reliable and effective disease screening methods are necessary for identification of resistance to plant pathogens among plant cultivars, breeding lines, and accessions. A reliable screening procedure facilitates accurate differentiation of resistant from susceptible germ plasm. Several techniques have been developed to test plants for resistance to Sclerotinia stem rot or white mold. Two screening methods, ascospore inoculation and limited-term inoculation, often used in Sclerotinia resistance assays, were compared in this study. Results within each inoculation method were consistent, and different levels of resistance among Capsicum spp. accessions were identified. Results of the ascospore inoculation tests concurred with the findings of $\mathrm{Tu}$ (22), who reported that ascospores infected prebloom bean plants without an exogenous nutrient base. The

Table 2. Mean incidence of Sclerotinia stem rot (percent stem rot and plant death) of seedlings of commercial pepper cultivars inoculated with ascospores of Sclerotinia sclerotiorum in a growth chamber $^{\mathrm{x}}$

\begin{tabular}{lccccc}
\hline & \multicolumn{2}{c}{ Stem rot } & & \multicolumn{2}{c}{ Plant death } \\
\cline { 2 - 3 } \cline { 5 - 6 } Cultivar & Percent $^{\mathbf{y}}$ & AUPDC $^{\mathbf{z}}$ & & Percent $^{\mathbf{y}}$ & AUDPC $^{\mathbf{z}}$ \\
\hline Big Bertha & $100 \mathrm{a}$ & $30 \mathrm{a}$ & & $100 \mathrm{a}$ & $26 \mathrm{a}$ \\
Astrione & $96 \mathrm{ab}$ & $28 \mathrm{a}$ & & $92 \mathrm{ab}$ & $24 \mathrm{a}$ \\
Camelot & $96 \mathrm{ab}$ & $29 \mathrm{a}$ & & $92 \mathrm{ab}$ & $25 \mathrm{a}$ \\
Lilac & $92 \mathrm{abc}$ & $29 \mathrm{a}$ & & $92 \mathrm{ab}$ & $25 \mathrm{a}$ \\
Pax 2557 & $92 \mathrm{abc}$ & $26 \mathrm{ab}$ & & $92 \mathrm{ab}$ & $23 \mathrm{a}$ \\
Marengo & $85 \mathrm{bc}$ & $28 \mathrm{a}$ & & $77 \mathrm{abc}$ & $21 \mathrm{ab}$ \\
Long Hungarian Wax & $84 \mathrm{bcd}$ & $22 \mathrm{abc}$ & & $80 \mathrm{bc}$ & $17 \mathrm{abc}$ \\
King Arthur & $81 \mathrm{bcd}$ & $19 \mathrm{abc}$ & & $77 \mathrm{abc}$ & $16 \mathrm{bc}$ \\
Tequila & $73 \mathrm{bcd}$ & $12 \mathrm{c}$ & & $70 \mathrm{bc}$ & $10 \mathrm{c}$ \\
ORI & $66 \mathrm{~cd}$ & $21 \mathrm{abc}$ & & $62 \mathrm{c}$ & $17 \mathrm{abc}$ \\
Tiara & $66 \mathrm{~cd}$ & $15 \mathrm{bc}$ & & $62 \mathrm{c}$ & $11 \mathrm{bc}$ \\
SS 860 & $51 \mathrm{~d}$ & $13 \mathrm{c}$ & & $51 \mathrm{c}$ & $10 \mathrm{c}$ \\
\hline
\end{tabular}

${ }^{\mathrm{x}}$ Means within a column followed by same letter are not significantly different according to Fisher's protected least significant difference test $(P \leq 0.05)$.

${ }^{y}$ Analysis of variance was performed on arcsin transformed data. Means of non-transformed data are presented.

${ }^{\mathrm{z}}$ AUDPC $=$ area under the disease progress curve.

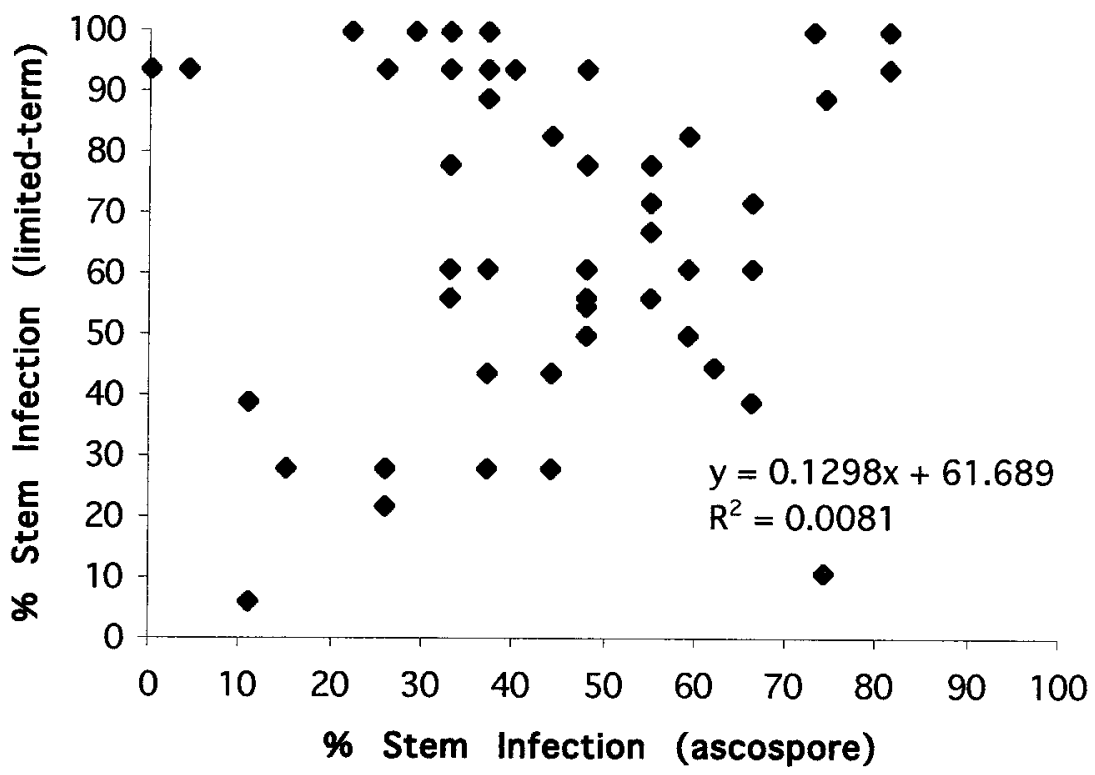

Fig. 1. Relationship of percentage of stem rot from ascospore inoculation and limited-term inoculation (LTI) tests for resistance of 51 accessions of Capsicum spp. tested under growth chamber conditions.

results do, however, contradict the results of other studies $(2,9,14,20)$, in which ascospores did not infect unless senescent tissues (flower petals) or wounds were present.

Factors involved in direct ascospore inoculation of pepper seedlings without a nutrient base (senescent flower petals or dead tissue) are not known. It is speculated

Table 3. Comparison of stem rot incidence for 51 Capsicum spp. accessions averaged over three tests after inoculation using ascospores or the limited-term method

\begin{tabular}{|c|c|c|}
\hline \multirow[b]{2}{*}{ Accession $^{\mathrm{z}}$} & \multicolumn{2}{|c|}{ Stem rot incidence $(\%)^{\mathrm{y}}$} \\
\hline & Ascospore & Limited-term \\
\hline PI 124080 & $81 \mathrm{a}$ & $94 \mathrm{ab}$ \\
\hline PI 164564 & $81 \mathrm{a}$ & $100 \mathrm{a}$ \\
\hline PI 164565 & $74 \mathrm{ab}$ & $89 a b c$ \\
\hline PI 159265 & $74 \mathrm{ab}$ & $11 \mathrm{ij}$ \\
\hline PI 164678 & $73 \mathrm{ab}$ & $100 \mathrm{a}$ \\
\hline PI 251622 & $66 \mathrm{abc}$ & 39 fghi \\
\hline PI 167062 & $66 a b c$ & $61 \mathrm{c}-\mathrm{g}$ \\
\hline PI 169110 & $66 \mathrm{abc}$ & $72 \mathrm{~b}-\mathrm{f}$ \\
\hline PI 184037 & 62 abcd & $45 \mathrm{efgh}$ \\
\hline PI 102882 & 59 abcd & 83 bcd \\
\hline PI 263073 & 59 abcd & $61 \mathrm{c}-\mathrm{g}$ \\
\hline PI 263106 & $59 \mathrm{abcd}$ & $50 \mathrm{~d}-\mathrm{h}$ \\
\hline PI 169106 & $55 \mathrm{a}-\mathrm{e}$ & 78 bcde \\
\hline PI 164961 & $55 \mathrm{a}-\mathrm{e}$ & 72 b-f \\
\hline PI 124540 & $55 \mathrm{a}-\mathrm{e}$ & $67 \mathrm{~b}-\mathrm{f}$ \\
\hline PI 167215 & $55 \mathrm{a}-\mathrm{e}$ & $56 \mathrm{c}-\mathrm{h}$ \\
\hline PI 215699 & $48 \mathrm{a}-\mathrm{f}$ & $50 \mathrm{~d}-\mathrm{h}$ \\
\hline PI 159278 & $48 \mathrm{a}-\mathrm{f}$ & $94 \mathrm{ab}$ \\
\hline PI 262905 & $48 \mathrm{a}-\mathrm{f}$ & $78 \mathrm{~b}-\mathrm{e}$ \\
\hline PI 184039 & $48 \mathrm{a}-\mathrm{f}$ & $61 c-g$ \\
\hline PI 249907 & $48 \mathrm{a}-\mathrm{f}$ & $55 \mathrm{~d}-\mathrm{h}$ \\
\hline PI 169112 & $48 \mathrm{a}-\mathrm{f}$ & $56 \mathrm{c}-\mathrm{h}$ \\
\hline PI 131352 & $44 \mathrm{~b}-\mathrm{g}$ & $83 \mathrm{bcd}$ \\
\hline PI 501532 & $44 \mathrm{~b}-\mathrm{g}$ & 28 ghij \\
\hline PI 164562 & $44 \mathrm{~b}-\mathrm{g}$ & $44 \mathrm{efgh}$ \\
\hline PI 260426 & $40 \mathrm{~b}-\mathrm{h}$ & $94 \mathrm{ab}$ \\
\hline PI 138561 & $37 \mathrm{c}-\mathrm{h}$ & $100 \mathrm{a}$ \\
\hline PI 169102 & $37 \mathrm{c}-\mathrm{h}$ & $89 a b c$ \\
\hline PI 123166 & $37 \mathrm{c}-\mathrm{h}$ & $89 a b c$ \\
\hline PI 289762 & $37 \mathrm{c}-\mathrm{h}$ & $61 \mathrm{c}-\mathrm{g}$ \\
\hline PI 162608 & $37 \mathrm{c}-\mathrm{h}$ & 28 ghij \\
\hline PI 127445 & $37 \mathrm{c}-\mathrm{h}$ & $94 \mathrm{ab}$ \\
\hline PI 103048 & $37 \mathrm{c}-\mathrm{h}$ & 44 efgh \\
\hline PI 135826 & $33 \mathrm{c}-\mathrm{h}$ & $100 \mathrm{a}$ \\
\hline PI 123474 & $33 \mathrm{c}-\mathrm{h}$ & $94 \mathrm{ab}$ \\
\hline PI 257049 & $33 \mathrm{c}-\mathrm{h}$ & $61 \mathrm{c}-\mathrm{g}$ \\
\hline PI 164561 & $33 \mathrm{c}-\mathrm{h}$ & $56 \mathrm{~d}-\mathrm{h}$ \\
\hline PI 152222 & $33 \mathrm{c}-\mathrm{h}$ & 78 bcde \\
\hline PI 102881 & $29 \mathrm{~d}-\mathrm{h}$ & $100 \mathrm{a}$ \\
\hline PI 148626 & $29 \mathrm{~d}-\mathrm{h}$ & $100 \mathrm{a}$ \\
\hline PI 162607 & $26 \mathrm{e}-\mathrm{i}$ & 28 ghij \\
\hline PI 235047 & $26 \mathrm{e}-\mathrm{i}$ & $22 \mathrm{hij}$ \\
\hline PI 257187 & $26 e-i$ & $94 a b$ \\
\hline PI 260595 & $22 \mathrm{e}-\mathrm{i}$ & $100 \mathrm{a}$ \\
\hline PI 297456 & $22 \mathrm{e}-\mathrm{i}$ & $100 \mathrm{a}$ \\
\hline PI 263074 & $15 \mathrm{ghi}$ & 28 ghij \\
\hline PI 260434 & $11 \mathrm{hij}$ & $39 \mathrm{f}-\mathrm{i}$ \\
\hline PI 511886 & $11 \mathrm{hij}$ & $6 \mathrm{j}$ \\
\hline PI 159255 & $11 \mathrm{hij}$ & $6 \mathrm{j}$ \\
\hline PI 152217 & $4 \mathrm{ij}$ & $94 \mathrm{ab}$ \\
\hline PI Grif 1567 & $0 j$ & $94 \mathrm{ab}$ \\
\hline
\end{tabular}

y Ascospore and limited-term inoculation. Data were transformed using arcsin transformation prior to analysis. Nontransformed data are shown. Means within a column followed by same letter are not significantly different according to Fisher's protected least significant difference $(P \leq 0.05)$.

${ }^{\mathrm{z}}$ Species of these accessions are listed in Table 1. 
that individual spots formed by germinating ascospores on leaves of susceptible cultivars or lines may stimulate a sufficient amount of exudates on the leaf surface for subsequent hyphal development and infection. Similarly, ascospores caused small necrotic lesions that occasionally developed into rapidly expanding, water-soaked lesions on field-grown clover (13) and greenhouse-grown soybean (20) without supplemental nutrients. Pepper seedlings produced under the conditions of this study were relatively succulent and may have provided exudates with a nutrient base adequate for pathogen growth and development. Succulent tissue may be more susceptible to direct infection by $S$. sclerotiorum than are hardened tissues. Symptoms on Capsicum spp. started as local brown spots on leaves of susceptible, as well as some resistant, plants. These spots then developed into water-soaked lesions in susceptible cultivars and accessions, which resulted in complete collapse of seedlings. Water-soaked lesions did not develop in resistant cultivars and accessions. Similar small leaf spot development has been reported in lettuce (15), bean and soybean (20), and sunflower (3) following ascospore inoculations.

There was no correlation between ascospore inoculation and LTI test results in this study. The LTI method was more severe than ascospore inoculation; some of the accessions having $30 \%$ or less stem rot after ascospore inoculation had $90 \%$ or greater stem rot in LTI tests.

C. baccatum var. pendulum accession PI 152217 had 4 and $94 \%$ stem rot when inoculated with an ascospore suspension and LTI, respectively. The opposite reaction also occurred occasionally. For example, C. annuum accession PI 159265 had 74 and $11 \%$ stem rot when inoculated with an ascospore suspension and LTI, respectively. All 12 commercial pepper cultivars were susceptible to Sclerotinia stem rot, regardless of inoculation method, although the plants died more quickly when LTI was used than when ascospores were the inoculum. The susceptible Capsicum spp. accessions also died more quickly using LTI than with ascospore inoculation. Inoculated plants from $C$. annuum accessions PI 159255 and PI 263074, and C. baccatum accession PI 260434 exhibited small brown spots on leaves and stems, which usually did not progress further, regardless of the inoculation method. These restricted spots or necroses may result from a hypersensitive response by the host to the pathogen in these accessions. Similar types of restricted lesions were reported on the soybean cultivars Corsoy, Williams, and Union when inoculated using the LTI method (10). $C$. galapagoense accession PI Grif 1567 showed no symptoms when inoculated with ascospores but was highly susceptible when tested using the LTI method. PI Grif 1567 has a hairy leaf surface, which may prevent ascospore attachment and penetration by the germ tube. This plant surface feature may have formed a physical barrier that prevented infection, thus protecting the plant. Inoculation of plants by wrapping the fungal mycelium around the stem may eliminate this barrier and also may injure the numerous trichomes on the stem, resulting in potential direct points of entry into the epidermal cells.

The results obtained in this study demonstrate for the first time that a high level of Sclerotinia stem rot resistance exists among the Capsicum spp. accessions. Resistance screening programs should, however, include both inoculation methods. Some of the most resistant accessions were C. annuum lines; therefore, barriers to hybridization associated with several of the species evaluated would not be an issue. Genetic resistance may be a feasible alternative for management of this very severe disease.

\section{ACKNOWLEDGMENTS}

We thank A. Dorrance and L. Rhodes for critical review of this manuscript.

\section{LITERATURE CITED}

1. Abawi, G. S., and Grogan, R. G. 1979. Epidemiology of diseases caused by Sclerotinia species. Phytopathology 69:899-904.

2. Abawi, G. S., Providenti, R., Crosier, D., and Hunter, J. E. 1975. Evaluating bean germplasm for resistance to Whetzelinia sclerotiorum. Proc. Am. Phytopathol. Soc. 2:50

3. Achbani, H., Tourvieille de Labrouhe, D., and Vear, F. 1996. Resistance of sunflower (Helianthus annuum L.) to terminal bud attack by Sclerotinia sclerotiorum de Bary. Eur. J. Plant Pathol. 102:421-429.

4. Aleksic, Z., and Aleksic, D. 1978. The most important diseases of pepper in Serbia. Rev. Plant Pathol. 57: 250.

5. Anonymous 1960. Index of Plant Diseases in the United States. U. S. Dep. Agric. Res. Ser. Div. Crops Res. Handb. No. 165.

6. Ben-Yephet, Y., and Bitton, J. 1985. Use of a selective medium to study the dispersal of ascospores of Sclerotinia sclerotiorum. Phytoparasitica 13:33-40.

7. Boland, G. J., and Hall, R. 1994. Index of plant hosts of Sclerotinia sclerotiorum. Can. J. Plant Pathol. 16:93-108.

8. Campbell, C. L., and Madden, L. V. 1990. Introduction to Plant Disease Epidemiology. Wiley-Interscience, New York.

9. Cline, M. N., and Jacobsen, B. J. 1983. Methods for evaluating soybean cultivars for resistance to Sclerotinia sclerotiorum. Plant Dis. 67:784-786.

10. Huang, H. C., and Kozub, G. C. 1989. A simple method for production of apothecia from sclerotia of Sclerotinia sclerotiorum. Plant Prot. Bull. 31:333-345.

11. Hunter, J. E., Dickson, M. H., Boettger, M. A., and Cigna, J. A. 1982. Evaluation of plant introductions of Phaseolus spp. for resistance to white mold. Plant Dis. 66:320-322.

12. Hunter, J. E., Dickson, M. H., and Cigna, J. A. 1981. Limited-term inoculation: A method to screen bean plants for partial resistance to white mold. Plant Dis. 65:414-417.

13. Loveless, A. R. 1951. Observation on the biology of clover rot. Ann. Appl. Biol. 38:642-664.

14. McLean, D. M. 1958. Role of dead flower parts in infection of certain Crucifers by Sclerotinia sclerotiorum (Lib.) de Bary. Plant Dis. Rep. 42:663-666.

15. Newton, H. C., and Sequeira, L. 1972. Ascospores as the primary infective propagules of Sclerotinia sclerotiorum in Wisconsin. Plant Dis. Rep. 56:798-802.

16. Raabe, R. D., Conners, I. L., and Martinez, A. P. 1981. Check list of plant diseases in Hawaii. Hawaii Inst. Trop. Agric. Human Res. Univ. Hawaii. Inf. Text Ser. No. 22.

17. Roy, A. K. 1974. New records of three diseases of chili in India. Indian Phytopathol. 27:620-622.

18. Srivastava, U. K., and Divacor, M. C. 1987. Note on stem rot and wilt of chilies in Haryana (India). Plant Prot. Bull. 39:35.

19. Steadman, J. R., and Cook, G. E. 1974. A simple method for collecting ascospores of Whetzelinia sclerotiorum. Plant Dis. Rep. 58:190.

20. Sutton, D. C., and Deverall, B. J. 1983. Studies on infection of bean (Phaseolus vulgaris) and soybean (Glycine max) by ascospores of Sclerotinia sclerotiorum. Plant Pathol. 32:251-261.

21. Tripathy, H., and Mahapatra, G. N. 1973. Host range of Sclerotinia sclerotiorum and Sclerotium rolfsii in Jordan. Assam Sci. Culture 39:319-320.

22. Tu, J. C. 1985. Tolerance of white bean (Phaseolus vulgaris) to white mold (Sclerotinia sclerotiorum) associated with tolerance to oxalic acid. Physiol. Plant Pathol. 26:111-117.

23. Weber, G. F. 1932. Disease of peppers in Florida. Florida Agric. Exp. Stn. Bull. 244.

24. Yanar, Y. 1997. Pathogenesis of Sclerotinia sclerotiorum (Lib.) de Bary on Pepper (Capsicum annuum L.). The Ohio State University, Columbus.

25. Yanar, Y., Sahin, F., and Miller, S. A. 1996. First report of stem and fruit rot of pepper caused by Sclerotinia sclerotiorum in Ohio. Plant Dis. 80:342. 